\title{
Differences between Breast Conservation-Eligible Patients and Unilateral Mastectomy Patients in Choosing Contralateral Prophylactic Mastectomies
}

\author{
Michael S. Sabel, MD, ${ }^{*}$ Casey T. Kraft, BS, ${ }^{\dagger}$ Kent A. Griffith, MS, MPH, ${ }^{\star}$ \\ Jessica M. Bensenhaver, MD, ${ }^{*}$ Lisa A. Newman, MD, MPH, ${ }^{*}$ Sarah T. Hawley, \\ $\mathrm{PhD}, \mathrm{MPH},{ }^{\S, \pi}$ and Adeyiza O. Momoh, $\mathrm{MD}^{\dagger}$
}

*Department of Surgery, University of Michigan Health System, Ann Arbor, Michigan; ${ }^{\dagger}$ Department of Plastic Surgery, University of Michigan Health System, Ann Arbor, Michigan; ${ }^{\star B i o s t a t i s t i c s}$

Core, University of Michigan Comprehensive Cancer Center, Ann Arbor, Michigan; ${ }^{\S}$ Department of Internal Medicine, University of Michigan Health System, Ann Arbor, Michigan; "School of Public Health, University of Michigan Health System, Ann Arbor, Michigan

- Abstract: There has been an increasing use of bilateral mastectomy (BM) for breast cancer. We sought to examine our trends among breast conservation (BCT) candidates and women recommended for unilateral mastectomy (UM). Our prospective breast cancer database was queried for women with a first-time, unilateral breast cancer. Patient and histologic factors and surgical treatment, including reconstruction, were evaluated. A detailed chart review was performed among patients from two representative time periods as to the reasons the patient underwent mastectomy. We identified 3,892 women between 2000 and 2012 of whom 60\% underwent BCT, 1092 (28\%) had UM and 12\% underwent BM. BM rose from $4 \%$ in 2000 to a high of $19 \%$ in 2011 , increasing around 2002 for women $<40$. BCT was less likely with decreasing age $(p<0.0001)$, lobular histology $(p<0.0001)$, higher stage $(p<0.0001)$ and decreasing BMI $(p<0.0001)$. Among mastectomy patients, contralateral mastectomy was associated with decreasing age $(p<0.0001)$, Caucasian race $(p<0.0001)$, and lower stage $(p=0.005)$. Over time, indications for mastectomy decreased while patients deemed BCT-eligible opting for UM or BM increased dramatically. Increases in the use of BM are in large part among women who were otherwise BCT-eligible. Factors associated with BM use are different for BCT-eligible patients and those recommended for UM. A better understanding of the factors driving individual patient choices is needed.

Key Words: breast cancer, breast conservation, contralateral prophylactic mastectomy, reconstruction

$\mathrm{O}$ ver the same period that we have seen a shift toward less aggressive axillary surgery in breast cancer, we have seen a parallel shift toward more aggressive breast surgery, with a significant increase in the rate of contralateral prophylactic mastectomy (CPM). This has been demonstrated in both single institution studies $(1,2)$, and in data from national databases (3-5). These trends occur despite the lack of evidence regarding the impact of CPM on survival, particularly among patients without a known increased risk for contralateral disease (6).

Address correspondence and reprint requests to: Michael S. Sabel, MD, Department of Surgery, University of Michigan Health System, 3302 Cancer Center, 1500 East Medical Center Drive, Ann Arbor, MI 48109, USA, or e-mail: msabel@umich.edu

DOI: $10.1111 /$ tbj.12648

(C) 2016 Wiley Periodicals, Inc., 1075-122X/16

The Breast Journal, Volume 22 Number 6, 2016 607-615
These trends have not occurred in a bubble. Over this same time period we have seen the increasing sensitivity of breast imaging, improvements in breast reconstruction, and our understanding of family history, genetics and risk. This information is not only available to clinicians, but is increasingly available to our patients via mass media, the internet and through social media. The institutional studies to date have primarily focused on patients undergoing mastectomy who then opted to also undergo CPM $(1,2)$. However, the threshold to undergo bilateral mastectomy (BM) when a unilateral mastectomy (UM) is necessary, is substantially different than opting for CPM when breast conservation (BCT) is an option. This study was performed to document trends in surgical therapies for all women with operable breast cancer, and identify those factors associated with both the decision to forego BCT as well as the decision to undergo BM. 


\section{METHODS}

All biopsy-proven breast cancer patients seen at the University of Michigan Comprehensive Cancer clinic are presented at a multidisciplinary tumor board composed of surgical, medical, radiation oncologists, radiologists, pathologists and associated support staff. Once the patient has undergone surgery, the data from these discussions and the patient treatment records are entered into our breast cancer database. With Institutional Review Board approval, our prospective breast cancer database was queried for all adult female patients 21 years of age and older who underwent surgery for a unilateral, primary stage I, II, or III breast cancer at the University of Michigan Comprehensive Cancer Center between January 1, 2000 and December 31, 2012. Women with bilateral cancers and women with a prior history of breast cancer were excluded from this study. The database was queried to obtain information regarding age at diagnoses, race/ethnicity, height and weight at diagnosis, and smoking history. Details of surgical treatment, including reconstruction, and tumor pathologic characteristics (tumor size, nodal status, grade, estrogen and progesterone receptor and HER2 status) were also collected. BMI was calculated using the Quetelet Index.

For select time-periods, a detailed review of the multidisciplinary visit was conducted to determine the tumor board recommendations, whether the patient opted for mastectomy, or the reason the patient was recommended to undergo mastectomy by the surgeon. For the purposes of this study, patient choice was defined as a clinical situation where the surgeon informed the patient that either BCT or mastectomy was an option, as documented at the initial multidisciplinary tumor board visit, and the patient chose to undergo mastectomy, either unilateral or bilateral. Tumor size, as a reason for recommending mastectomy, was defined as any situation where the size of the tumor (including surrounding calcifications), compared to the size of the breast was such that the surgeon felt the cosmesis would be poor, and thus mastectomy was a better option. This also included patients for whom neoadjuvant chemotherapy wasn't an option, or the tumor failed to decrease with neoadjuvant chemotherapy. BCT failure included any patient where BCT was attempted, but ultimately a mastectomy was performed, even if patients had an option for a re-excision lumpectomy. Multicentric disease was defined as more than one site of documented disease that could not be incorporated into a single lumpectomy. This did not include patients with suspicious areas on imaging who opted not to undergo biopsy, as they had opted for mastectomy. Additional reasons for mastectomy included patients with diffuse calcifications and those who could not undergo radiation, either secondary to prior nonbreast cancer radiation (as prior breast cancers were excluded), pregnancy, or collagen-vascular disease. Specific BRCA mutation status was not available in our database for analysis as part of this study.

The distribution of categories was compared using the chi-squared test statistic between lumpectomy and mastectomy groups, and between unilateral and bilateral groups within those having mastectomies. P-values at or below 0.05 were considered significant.

\section{RESULTS}

After excluding patients with a prior history of breast cancer or bilateral cancers, we identified 3,892 women with primary, unilateral breast cancer treated between 2000 and 2012. During this time period, $2,325(60 \%)$ underwent BCT, 1,092 (28\%) had a UM and $475(12 \%)$ underwent a BM. Table 1 shows the demographics and tumor characteristics and their association with undergoing BCT, UM, and BM. Younger age was a highly significant predictor of undergoing not only mastectomy, but also opting for CPM ( $\mathrm{p}<0.0001$ for both). This was particularly true among women under age 40, where only one-third of women opted for BCT. The proportion rose to over one-half of women age 40-49, and continued to rise as women aged. Race was not a factor significantly associated with BCT rates $(p=0.09)$. A similar percentage of white and African-American patients underwent BCT. However, race was strongly associated with undergoing $\mathrm{BM}$ as opposed to $\mathrm{UM}$, with white women having CPM at a significantly higher rate $(33 \%)$ than African American (18\%), Asian $(10 \%)$, or other races $(22 \%)(p<0.0001)$.

Overall, there was no difference in BCT rates or CPM usage for patients with in situ disease versus invasive disease. Among patients with invasive cancer, there was a significantly lower rate of BCT in patients with lobular carcinoma compared with ductal carcinoma $(48 \%$ versus $61 \%)$. Patients with "ductal with lobular features" and other histologies had a similar BCT rate to ductal carcinoma. Even though patients with lobular carcinoma were more likely to undergo 
Table 1. Factors Associated with Surgical Decisions among Patients with First-Time, Primary, Unilateral Breast Cancer

\begin{tabular}{|c|c|c|c|c|c|c|c|}
\hline & \multicolumn{2}{|c|}{ Breast conservation } & \multicolumn{2}{|c|}{ Unilateral mastectomy } & \multicolumn{2}{|c|}{ Bilateral mastectomy } & \multirow[b]{2}{*}{$\%$ of mastectomies } \\
\hline & $N$ & $\%$ of total & $N$ & $\%$ of total & $N$ & $\%$ of total & \\
\hline \multicolumn{8}{|l|}{ Age } \\
\hline$<40$ & 120 & 34 & 118 & 33 & 117 & 33 & 50 \\
\hline $40-49$ & 520 & 53 & 306 & 31 & 161 & 16 & 34 \\
\hline $50-59$ & 720 & 62 & 305 & 26 & 129 & 11 & 30 \\
\hline $60-69$ & 570 & 67 & 219 & 26 & 60 & 7 & 22 \\
\hline 70-79 & 299 & 73 & 110 & 27 & 3 & 1 & 3 \\
\hline$>80$ & 95 & 71 & 34 & 25 & 5 & 4 & 13 \\
\hline$p$-value & & & & & & $<0.0001$ & $<0.0001$ \\
\hline \multicolumn{8}{|l|}{ Race } \\
\hline White & 1,970 & 60 & 867 & 27 & 429 & 13 & 33 \\
\hline African American & 173 & 59 & 97 & 33 & 22 & 8 & 18 \\
\hline Asian & 79 & 50 & 70 & 45 & 8 & 5 & 10 \\
\hline Other & 103 & 58 & 58 & 33 & 16 & 9 & 22 \\
\hline$p$-value & & & & & & 0.0915 & $<0.0001$ \\
\hline \multicolumn{8}{|l|}{ Invasive versus in situ } \\
\hline In situ & 478 & 61 & 205 & 26 & 102 & 13 & 33 \\
\hline Invasive & 1,847 & 59 & 887 & 29 & 373 & 12 & 30 \\
\hline & 2,325 & & 1,092 & & 475 & & \\
\hline$p$-value & & & & & & 0.4607 & 0.2157 \\
\hline \multicolumn{8}{|l|}{ Histology } \\
\hline Ductal & 1,401 & 61 & 617 & 27 & 264 & 12 & 30 \\
\hline Lobular & 174 & 48 & 135 & 37 & 53 & 15 & 28 \\
\hline Ductal with lobular features & 146 & 59 & 76 & 31 & 26 & 10 & 25 \\
\hline Other & 112 & 61 & 49 & 27 & 23 & 13 & 32 \\
\hline p-value & & & & & & $<0.0001$ & 0.7430 \\
\hline \multicolumn{8}{|l|}{ T-stage } \\
\hline T1mic & 42 & 62 & 25 & 37 & 1 & 1 & 4 \\
\hline T1a & 174 & 64 & 67 & 24 & 33 & 12 & 33 \\
\hline T1b & 421 & 74 & 90 & 16 & 55 & 10 & 38 \\
\hline T1c & 716 & 67 & 246 & 23 & 107 & 10 & 30 \\
\hline T2 & 399 & 50 & 290 & 36 & 111 & 14 & 28 \\
\hline T3 & 24 & 16 & 95 & 64 & 30 & 20 & 24 \\
\hline$p$-value & & & & & & $<0.0001$ & 0.0058 \\
\hline \multicolumn{8}{|l|}{ Node positivity } \\
\hline No & 1,380 & 68 & 442 & 22 & 208 & 10 & 32 \\
\hline \multirow[t]{2}{*}{ Yes } & 460 & 43 & 446 & 42 & 165 & 15 & 27 \\
\hline & 1,840 & & 888 & & 373 & & \\
\hline$p$-value & & & & & & $<0.0001$ & 0.0521 \\
\hline \multicolumn{8}{|l|}{ BMI } \\
\hline$<20$ & 66 & 46 & 51 & 35 & 27 & 19 & 35 \\
\hline $20-24.9$ & 514 & 55 & 265 & 28 & 155 & 17 & 37 \\
\hline $25-29.9$ & 559 & 58 & 283 & 30 & 114 & 12 & 29 \\
\hline $30-34.9$ & 343 & 63 & 147 & 27 & 57 & 10 & 28 \\
\hline $35-39.9$ & 194 & 66 & 67 & 23 & 31 & 11 & 32 \\
\hline $40-44.9$ & 83 & 66 & 26 & 21 & 17 & 13 & 40 \\
\hline \multirow[t]{2}{*}{$>45$} & 77 & 68 & 26 & 23 & 10 & 9 & 28 \\
\hline & 1,836 & & 865 & & 411 & & \\
\hline$p$-value & & & & & & $<0.0001$ & 0.1349 \\
\hline \multicolumn{8}{|l|}{ Tobacco use } \\
\hline Yes & 590 & 62 & 267 & 28 & 96 & 10 & 26 \\
\hline No & 1,586 & 59 & 757 & 28 & 347 & 13 & 31 \\
\hline Unknown & 127 & 60 & 59 & 28 & 25 & 12 & 30 \\
\hline$p$-value & & & & & & 0.2773 & 0.1990 \\
\hline
\end{tabular}

mastectomy, this was not a factor associated with choosing to undergo BM, with a similar fraction to other invasive histologies $(\mathrm{p}=0.734)$.

Among patients with invasive disease, tumor size was, as expected, associated with decreasing use of
BCT, but not removal of the contralateral breast. In fact, increasing T-stage was associated with a decreased likelihood of undergoing contralateral mastectomy $(p=0.006)$. A similar pattern was seen with nodal involvement. Patients with node positive disease 

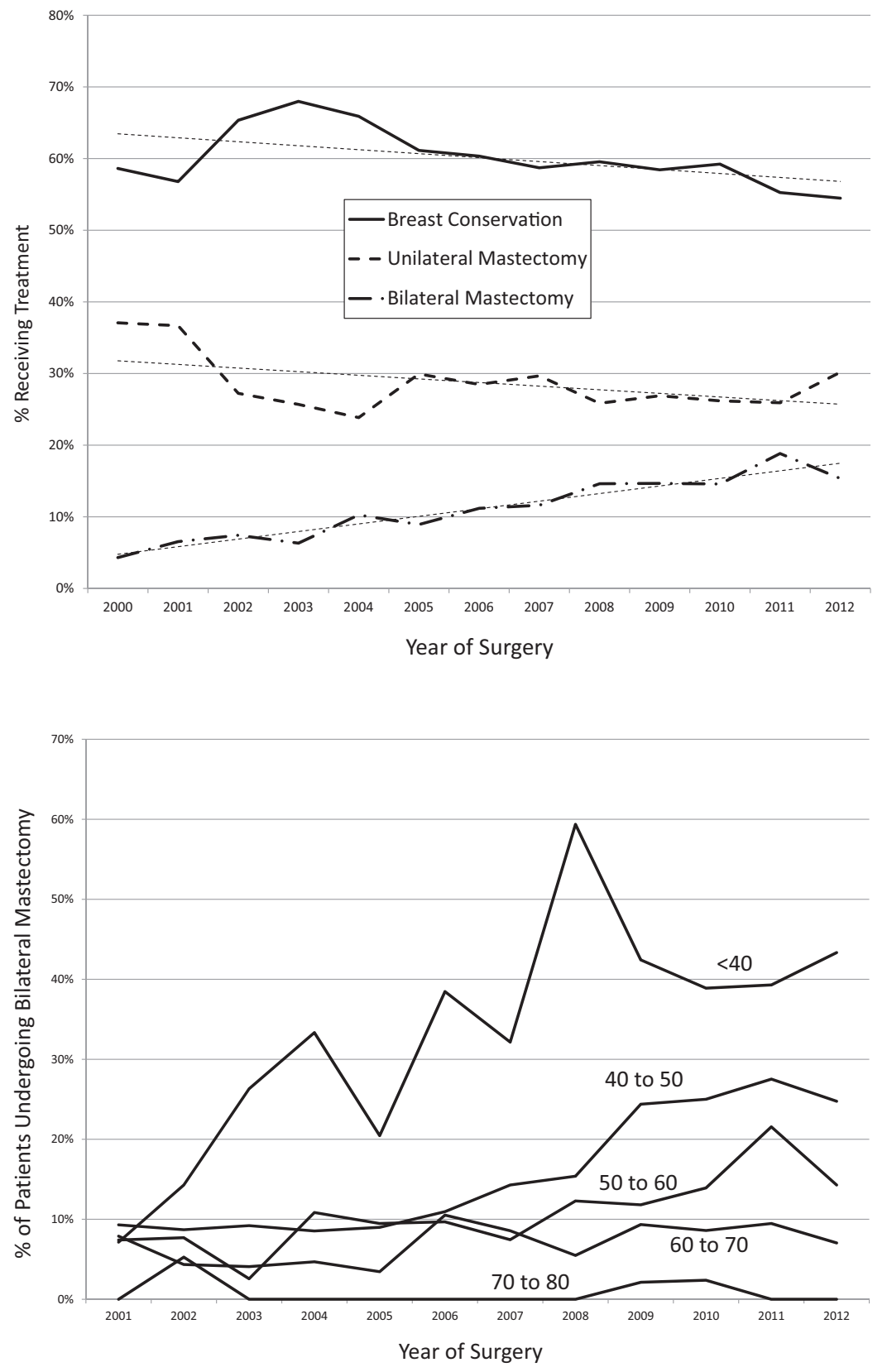

Figure 1. Trends in breast conservation, unilateral and bilateral mastectomy among patients with unilateral, primary breast cancer.

Figure 2. Trends in patients with unilateral, primary breast cancer undergoing bilateral mastectomy by decade. While bilateral mastectomy has risen among women $<40$ since 2002, it began increasing for women between 40 and 60 around 2008.

were less likely to have BCT $(43 \%$ versus $68 \%$, $\mathrm{p}<0.0001$ ), but less likely to have contralateral mastectomies $(27 \%$ versus $32 \%, \mathrm{p}=0.05)$.

The year of treatment was strongly associated with surgical decision making (Fig. 1). Over the period from 2000 through 2012, we saw a decrease in the BCT rate, ranging from a high of $68 \%$ in 2003 to a low of $54 \%$ in 2012. During this time period, the UM rate remained relatively constant while the $\mathrm{BM}$ increased, from a low of $4 \%$ in 2000 to a high of $19 \%$ in 2011 . The rise in bilateral mastectomies was most significant among women less than age 40
(Fig. 2), however there were also rises among women in their forties and fifties. These appear to have increased at several years later, with the increase in women $<40$ starting around 2002, but not increasing for women 40-60 until around 2007 or 2008.

\section{RECONSTRUCTION}

As improved reconstruction options have been implicated as a reason women increasingly opt to undergo BM, we examined these trends. Over this entire time period, patients undergoing mastectomy 
Figure 3. Trends in immediate or delayed reconstruction after mastectomy.

Figure 4. Trends in patients undergoing reconstruction after unilateral or bilateral mastectomy.
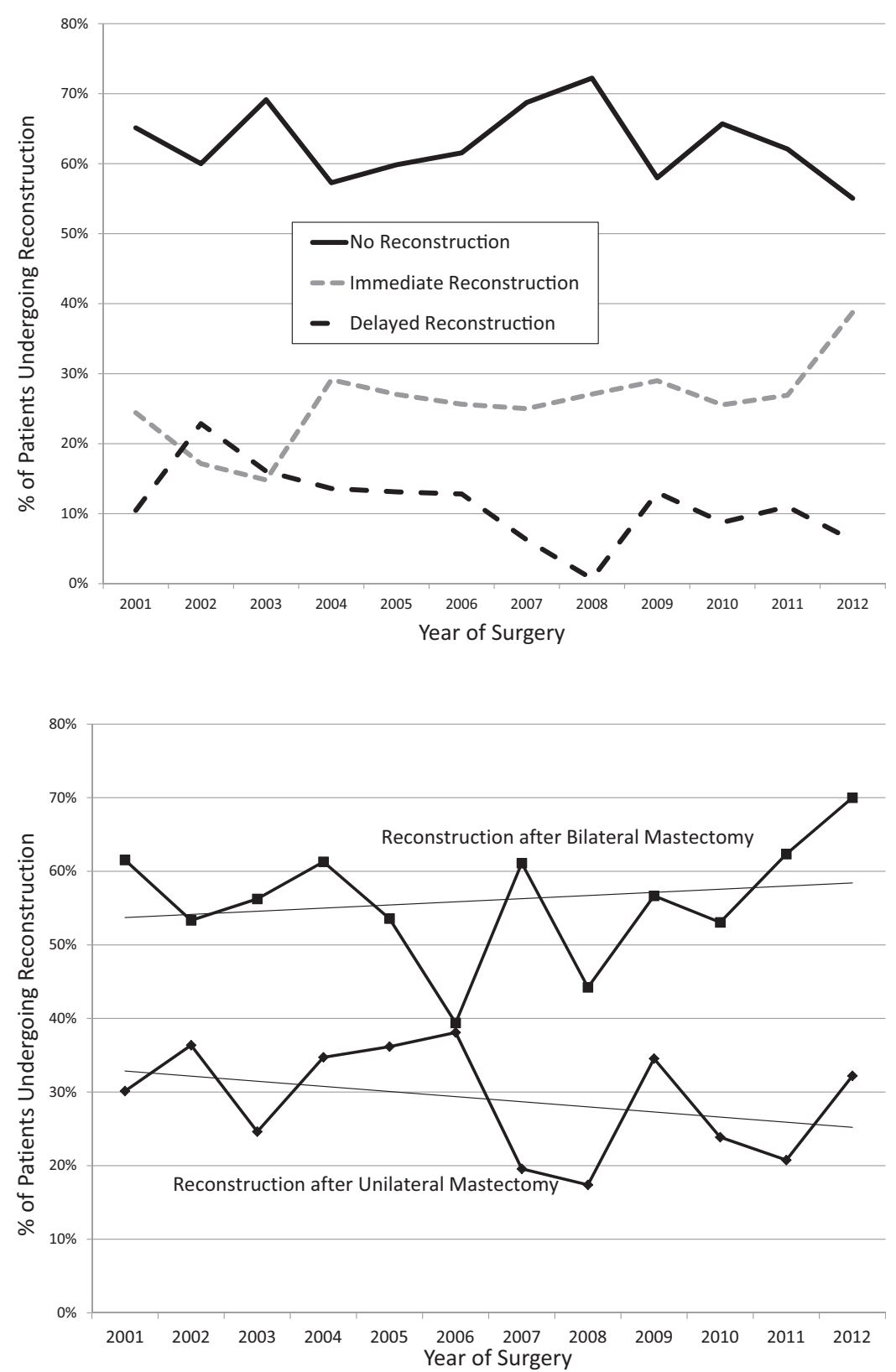

were offered consultation with plastic surgery to discuss reconstructive options. Among the 1,565 patients undergoing unilateral or BM, $428(27 \%)$ opted to have immediate reconstruction and another 161 $(10 \%)$ underwent delayed reconstruction. The most common form of reconstruction was with implants, with or without expanders $(71 \%)$, while autologous reconstruction represented $29 \%$, with the most common technique being a pedicled transverse rectus abdominis myocutaenous (TRAM) flap (17\%).

Overall, from 2000 through 2012, although mastectomy rates increased, we did not see an overall change in the percentage of mastectomy patients who opted for reconstruction, fluctuating between $24 \%$ and $31 \%$ of patients undergoing either unilateral or $\mathrm{BM}(\mathrm{p}=\mathrm{NS})$. Over this time period, however, we did see a shift from delayed reconstruction to immediate reconstruction (Fig. 3). While throughout the years of this study the ratio of allogeneic versus autologous reconstruction remained constant, the type of autologous reconstruction changed, with pedicled TRAM flaps dropping to a smaller percentage of autologous reconstructions in favor of muscle sparing TRAMs and deep inferior epigastric perforator flaps. 


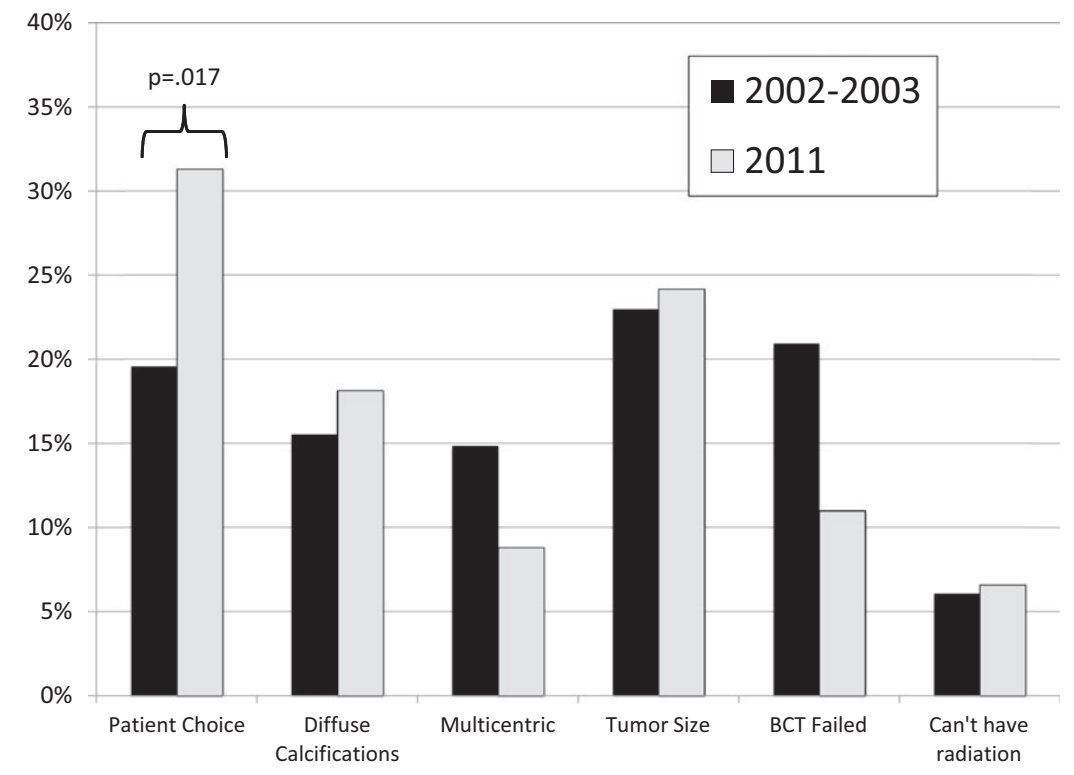

Figure 5. Reasons for undergoing mastectomy in 2002-2003 and 2011. Patient choice includes all patients for whom breast conservation was an option but the patient chose unilateral or bilateral mastectomy.
Breaking down mastectomy patients by unilateral versus bilateral, there has also been no change over time in the fraction of patients undergoing reconstruction over time for either group. Reconstruction was consistently more common among women undergoing BM than unilateral reconstruction (Fig. 4).

We also examined additional factors that may impact a woman's suitability to undergo reconstruction, and hence their decision to undergo mastectomy, including tobacco use and BMI. These data are shown in Table 1. Patients with a current or recent smoking history (37\% of patients), did not have a higher rate of mastectomy compared with nonsmokers, and there was no impact of smoking history on bilateral versus unilateral mastectomies $(\mathrm{p}=0.27$ and 0.19 respectively). Increasing BMI did impact surgical decision making, with BCT increasing as BMI increased $(\mathrm{p}<0.0001)$. However, among women undergoing mastectomy, there was no correlation between BMI and the decision to undergo bilateral versus UM $(\mathrm{p}=0.13)$.

\section{REASONS FOR MASTECTOMY}

As the threshold for undergoing BM is lower when a UM is recommended, we examined whether the rise in CPM may be related to increased indications for UM. To do this, we reviewed the charts for the 452 patients treated in 2002-2003, when the BCT rate was at its highest $(67 \%)$, and the 408 patients treated in the year 2011 (when BCT was at 55\%). Using medical record data as described above, we differentiated between those situations where the surgeon recommended mastectomy and those situations where the patient was felt to be a candidate for BCT by the surgeon (and was offered both choices), and opted to pursue mastectomy (unilateral or bilateral). Figure 5 shows the differences between these two time periods. Over this time period, there were minimal differences in the reasons women were recommended to undergo mastectomy, with no statistically significant difference between diffuse calcifications, tumor size, multicentricity or inability to have radiation. In fact, there was a small decrease in the percentage of patients recommended to undergo mastectomy, specifically after a failed attempt at BCT, secondary to our incorporation of intraoperative margin analysis in 2009 (7).

In contrast, there was a dramatic and statistically significant increase in the number of patients who were eligible for BCT, but instead opted to undergo mastectomy, rising from $19.6 \%$ in $2002-2003$ to $31.3 \%$ in $2011(\mathrm{p}=0.0169)$. The addition of a CPM among these patients also rose, both for patients requiring mastectomy (13-33\%) and for patients opting for mastectomy, which was nearly half $(45 \%)$ of patients in 2002-2003, and rose to nearly $2 / 3 \mathrm{rds}$ $(63 \%)$ in 2011.

\section{DISCUSSION}

At the University of Michigan we have witnessed a similar increasing trend in the use of the CPM as has 
been described nationally. This is not, however, an increasing use of CPM among patients recommended to undergo mastectomy, but rather is associated with a corresponding drop in BCT rates. This is despite a strong institutional bias toward BCT. The use of bilateral mastectomies rose from $4 \%$ to a high of $19 \%$ and was strongly associated with young age, as only one-third of patients under the age of 40 and one-half of patients between 40 and 49 opted for BCT. The trend is not explained by changes in the ages of our patient population, as neither the median age nor the proportion of patients under 40 has changed over this time period.

Several articles have described the increased popularity of the contralateral mastectomy, both at single institutions and in national databases (1-5). When simply tracking whether a patient had a CPM or not, it can sometimes be difficult to discern whether this represents women who are undergoing a UM and have opted to remove the other breast (for reasons that might not be related to risk reduction), or these are women who were excellent candidates for BCT, and opted for the more radical procedure primarily for prophylaxis. Examining the CPM rate in the context of all surgical decision making during this time period, and excluding patients with bilateral cancer and prior breast cancers (as this might influence decision making), we note both a slight decrease in the number of patients undergoing UM, and a decrease in the number of patients undergoing BCT. Although this may reflect a change in referral patterns, it suggests that this pattern is not just women undergoing mastectomy opting to have both breasts removed. Instead, a substantial component of women undergoing BM today were patients who 10 years ago would have undergone BCT (8). This is confirmed by recent data from the National Cancer Database which shows a small decrease in lumpectomy rates between 2003 and 2010 (5).

Several factors have been associated with a higher likelihood of undergoing a CPM. Our results suggest there is a difference between those factors that may be associated choosing BM among all patients, and those factors associated with opting for a contralateral mastectomy when committed to a UM. For example, race has been strongly associated with surgical decision making, with minorities less often getting CPM $(2,9,10)$. Our data show that overall, BCT rates were extremely similar among the races, however, white women, compared with other races, were more likely to undergo $\mathrm{BM}$ compared with UM, a finding consistent with other studies of CPM in different samples/settings $(11,12)$. Histology showed the opposite trend. There was no difference in BCT or BM rates between in situ and invasive disease. Lobular histology was associated with a significantly lower BCT rate (potentially due to tumor size or failure to achieve negative margins) Women with lobular histology undergoing mastectomy were not more likely to undergo BM, despite the perceived association with an increased risk of contralateral disease, and in contrast with studies showing increased CPM among women with lobular histology $(9,10)$. Increasing tumor size expectedly correlates with mastectomy over BCT. However, larger tumors are associated with a decreased likelihood of BM. In a similar vein, nodal positivity has a positive association with mastectomy, but a negative association with BM. As any potential benefit of prophylactic surgery decreases as the risk of recurrence of the known cancer rises, these trends may reflect appropriate counseling.

Age is by far the most significant factor for both, in our study and others $(1-3,9,10,13)$. Younger individuals may have more anxiety regarding recurrence and second primary tumors in their lifetime, and are more likely to harbor a genetic predisposition. Genetic testing, and the increased identification of patients carrying the BRCA1 and BRCA2 mutations, has certainly contributed to this trend. In 2002, the turnaround time for obtaining genetic testing results dropped significantly, allowing for preoperative counseling and shifting genetic testing from survivors to newly diagnosed patients. This may explain a jump in prophylactic mastectomies prior to 2002, but over this time period, and in the two specific time periods chosen for comparison (2002-2003 and 2011), BRCA testing was readily available. Though we were not able to identify BRCA mutation status in our patients, we do know that testing became easier for patients to obtain, and the guidelines for genetic testing were broadened, potentially identifying a slightly larger subset of patients harboring a genetic predisposition. Thus we may assume that more patients were tested and this may have contributed to a fraction of CPM. However, the analysis examining the reason for choosing mastectomy was abstracted from the initial conversation with the surgeon after multidisciplinary presentation, at which point BRCA mutation status was rarely known. Most patients informed the surgeon of their decision to proceed with $\mathrm{BM}$ before referral to the genetic counselor or testing. 
Breast imaging has changed considerably over this time period with the introduction of digital mammography and breast MRI. At the University of Michigan, we do not recommend routine breast MRI for BCT eligible patients, although an increasing number of patients arrive with MRIs obtained by outside physicians (radiologists, primary care physicians) prior to their initial consultation. Despite this, when one examines the reasons for patients undergoing mastectomy, diffuse calcifications, tumor size and multicentric disease (factors associated with sensitivity of imaging), did not significantly change.

In addition to increasing rates of CPM, there have been increasing rates of reconstruction and it has been hypothesized that the availability of reconstruction may be driving the mastectomy and BM rate $(14,15)$. Certainly, there may be some cases where mastectomy and reconstruction can achieve a better cosmetic outcome than BCT, and potentially avoid the complications of radiation. However, while reconstruction has been statistically linked to CPM rates, this does not necessarily translate to a causative relationship. At the University of Michigan, we have offered consultation with plastic surgery for any patient considering mastectomy for all the years included in this study. Although we have seen dramatic changes (more free flaps, a shift to more immediate reconstruction), we have not seen a rise in reconstruction rates among women undergoing either unilateral or bilateral reconstruction. In addition, factors that may impact the ability to undergo reconstruction, such as obesity and tobacco use, were not associated with the surgical decision making. These data suggest that while reconstruction rates may be increasing, changes in availability or morbidity are not, in large measure, driving BCT-eligible women toward either mastectomy or BM. Rather, women opting for mastectomy and CPM are doing so for other reasons, and then choosing whether to have reconstruction.

This of course begs the question why women are choosing more extensive surgery despite increased recovery time, complications and no clear benefit to survival (16). Although retrospective in nature, this single institution study does benefit from a consistency in practice over the time period being examined. Despite a pro-BCT approach, consistent use of genetic counseling/testing, consistent discussion of reconstruction options and referral to plastic surgery, avoidance of breast MRI and no significant change in patient age, BMI or presenting tumor stage, we too have seen the same concerning increase in bilateral mastectomies, particularly among younger women and even when limited to BCT-eligible patients.

What has increased over this time period, for which we have little control, is the public perception regarding the risk of a second cancer, the importance of family history, and the availability of prophylactic surgery. Surveys of women opting for CPM have identified a substantial fear of recurrence and a desire to prevent metastases and improve survival as quoted reasons $(11,12)$. However, many of these patients overestimate their risk for either dying of disease or developing a 2nd primary cancer, or misinterpret the benefit of BM (11,17-19). The perceived benefit of BM may be, to some degree, media driven. This has often been referred to as the "Angelina Jolie effect," a fear that interest in genetic testing and bilateral mastectomies would rise after the actress announced publicly that she had undergone risk reduction surgery. However, Angelina Jolie's announcement was in May of 2013, and the rise in bilateral mastectomies clearly began at least a decade prior to this. More importantly, the news coverage regarding Angelina Jolie's breast cancer centered on her family history and her BRCA status. In contrast, there have been many media reports of celebrities with breast cancer undergoing $\mathrm{BM}$ and immediate reconstruction, without explaining the decision making process or discussing alternative options.

In addition to this media attention, there is likely increased word of mouth as there are more breast cancer survivors and an increased willingness (if not encouragement) to talk about it. And while the risk of a contralateral cancer is low, estimated to be no more than $4-5 \%$ over 10 years $(20,21)$, given the prevalence of breast cancer, may be a large number of BCT patients who reply, "if I had to do it all over again, I'd have bilateral mastectomies," when queried about their decision; more when one includes patients dissatisfied with their cosmetic outcome.

While we clearly need better patient education regarding risk and the true benefits and complications of BM, the fact that higher education level seems to correlate with opting for CPM suggests that education alone may not be sufficient (22). It is clear that we need additional studies addressing the reasons why women choose BM, particularly those who are seemingly excellent candidates for BCT. A more nuanced approach to identifying and addressing patient fears and other motivating factors may be needed to 
counter the preconceived notions and mixed messages originating from outside sources.

\section{DISCLOSURE}

There was no financial or material support for this research, and the authors have no financial disclosures.

\section{REFERENCES}

1. Jones NB, Wilson J, Kotur L, et al. Contralateral prophylactic mastectomy for unilateral breast cancer: an increasing trend at a single institution. Ann Surg Oncol 2009;16:2691-6.

2. King TA, Sakr R, Patil S, et al. Clinical management factors contribute to the decision for contralateral prophylactic mastectomy. J Clin Oncol 2011;29:2158-64.

3. Yao K, Winchester DJ, Czechura T, Huo D. Contralteral prophylactic mastectomy and survival: report from the National Cancer Database, 1998-2002. Breast Cancer Res Treat 2013;142:465-76.

4. Tuttle TM, Habermann EB, Grund EH, et al. Increasing use of contralateral prophylactic mastectomy for breast cancer patients: a trend toward more aggressive surgical treatment. J Clin Oncol 2007;25:5203-9.

5. Pesce CE, Leiderbach E, Czechura T, et al. Changing surgical trends in young patients with early stage breast cancer, 2003 to 2010: a report from the national cancer database. JACS 2014;219:19-30.

6. Kurian AW, Lichtensztain DY, Keegan TH, et al. Use of and mortality after bilateral mastectomy compared with other surgical treatments for breast cancer in California, 1998-2011. JAMA 2014;12:902-14.

7. Sabel MS, Jorns JM, Wu A, Myers J, Newman LA, Breslin TM. Development of an intraoperative pathology consultation service at a free-standing ambulatory surgical center: clinical and economic impact for patients undergoing breast cancer surgery. Am J Surg 2012;204:66-77.

8. Dragun AE, Pan J, Riley EC, et al. Increasing use of elective mastectomy and contralateral prophylactic surgery among breast conservation candidates: a 14-year report from a comprenehensive cancer center. Am J Clin Oncol 2013;36:375-80.

9. Yi M, Hunt KK, Arun BK, et al. Factors affecting the deciion of breast cancer patients to undergo contralateral prophylactic mastectomy. Cancer Prev Res 2010;3:1026-34.
10. Arrington AK, Jarosek SL, Virnig BA, et al. Patient and surgeon characteristics associated with increased use of contralateral prophylactic mastectomy in patietns with breast cancer. Ann Surg Oncol 2009;16:2697-704.

11. Rosenberg SM, Tracy MS, Meyer ME, et al. Perceptions, knowledge and satisfaction with contralateral prophylactic mastectomy among young women with breast cancer. Ann Intern Med 2013;159:373-81.

12. Hawley ST, Jagsi R, Morrow M, et al. Social and clinical determinants of contralateral prophylactic mastectomy. JAMA Surg 2014;149:582-9.

13. Guth U, Myrick ME, Viehl CT, et al. Increasing rates of contralateral prophylactic mastectomy- a trend made in USA? Eur J Surg Oncol 2012;38:296-301.

14. Ashfaq A, McGhan LJ, Pockaj BA, et al. Impact of breast reconstruction on the decision to undergo contralateral prophylactic mastectomy. Ann Surg Oncol 2014;21:2934-40.

15. Jagsi R, Jiang J, Momoh AO, et al. Trends and variation in use of breast reconstruction in patients with breast cancer undergoing mastectomy in the United States. J Clin Oncol 2014;32:919-26.

16. Osman F, Saleh F, Jackson TD, et al. Increased postoperative complications in bilateral mastectomy patients compared to unilateral mastectomy: an Analysis of the NSQIP database. Ann Surg Oncol 2013;20:3212-7.

17. Black WC, Nease RF, Tosteson AN. Perceptions of breast cancer risk and screening effectiveness in women younger than 50 years of age. J Natl Cancer Inst 1995;87:720-31.

18. Rakovitch E, Franssen E, Kim J, et al. A comparison of risk perception and psychological morbidity in women with DCIS and early invasive breast cancer. Breast Cancer Res Treat 2003;77: 285-93.

19. Abbott A, Rueth N, Pappas-Varco S, et al. Perceptions of contralateral breast cancer: an oversestimation of risk. Ann Surg Oncol 2011;18:3129-36.

20. Davies C, Godwin J, Gray RJ, et al. Relevance of breast cancer hormone receptors and other factors to the effiacy of adjuvant tamoxifen: patient-level meta-analysis of randomised trials. Lancet 2011;378:771-84.

21. Nichols HB, Berrington de Gonzalez A, Lacey JV Jr, et al. Declining incidence of contralateral breast cancer in the United States from 1975 to 2006. J Clin Oncol 2011;29:1564-9.

22. Hawley ST, Jagsi R, Morrow M, et al. Social and clinical determinants of contralateral prophylactic mastectomy. JAMA Surg 2014;149:582-9. 